\title{
Role of maximum standardized uptake value in fluorodeoxyglucose positron emission tomography/computed tomography predicts malignancy grade and prognosis of operable breast cancer: a multi-institute study
}

\author{
Takayuki Kadoya $\cdot$ Kenjiro Aogi $\cdot$ Sachiko Kiyoto $\cdot$ \\ Norio Masumoto $\cdot$ Yoshifumi Sugawara • \\ Morihito Okada
}

Received: 13 August 2013/Accepted: 27 August 2013/Published online: 13 September 2013

(C) The Author(s) 2013. This article is published with open access at Springerlink.com

\begin{abstract}
The malignant biological behavior of breast cancer remains obscure on diagnostic images, although understanding the grade of such malignancy is important for selecting appropriate treatment. Therefore, malignancy grades in operable breast cancer were evaluated using positron emission tomography/computed tomography (PET/CT) in a multicenter setting. We prospectively examined the features and prognosis of 344 patients (mean age $\pm \mathrm{SD}$ : $58.0 \pm 12.5$ years) with clinical stages I-III breast cancer, who underwent surgical intervention without induction therapy between January 2006 and December 2011. Maximum standardized uptake values (maxSUV) obtained from wholebody fluorodeoxyglucose-PET/CT immediately before surgery were assessed to predict the malignant aggressiveness of tumors including the recurrence-free survival of the patients. Variations in maxSUV among institutions, which are limitations of PET assessments in multicenter studies, were adjusted using a phantom study. The median follow up period was 52 months. The patients were divided into groups
\end{abstract}

T. Kadoya · N. Masumoto · M. Okada ( $\square)$

Department of Surgical Oncology, Research Institute for

Radiation Biology and Medicine, Hiroshima University,

1-2-3-Kasumi, Minami-ku, Hiroshima, Hiroshima 734-0037,

Japan

e-mail: morihito@hiroshima-u.ac.jp

T. Kadoya

e-mail: takayukikadoya@gmail.com

\section{K. Aogi}

Department of Breast Surgery, Shikoku Cancer Center,

Matsuyama, Japan

S. Kiyoto · Y. Sugawara

Department of Radiation Therapy, Shikoku Cancer Center,

Matsuyama, Japan according to cut-off maxSUV $(\leq 3.0$ vs. $>3.0)$ values established from receiver operating characteristic analysis of recurrence (area under the curve $=0.713$ ). A higher maxSUV was significantly associated with a higher T-factor $(p<0.0001)$, $\mathrm{N}$-factor $(p=0.0049)$, nuclear grade $(p<0.0001)$, negative for estrogen $(p=0.0309)$, and progesterone receptors $(p=0.0063)$, positive for human epidermal growth factor receptor $2(p=0.0012)$, lymph node metastasis $(p=0.0128)$, and vascular invasion $(p=0.0110)$. Multivariate analysis using Cox proportional hazard regression model revealed high maxSUV and negative estrogen receptor status as significantly prognostic factors ( $p=0.033$ and $p=0.004$, respectively). This study demonstrated that maxSUV on PET/CT as well as estrogen receptor status is useful to predict malignancy grades and the prognosis of patients with breast cancer.

Keywords Breast cancer - PET/CT - maxSUV .

Prognosis - Estrogen receptor $\cdot$ Recurrence

$\begin{array}{ll}\begin{array}{l}\text { Abbreviations } \\ \text { FDG PET/CT }\end{array} & \begin{array}{l}\text { Fluorodeoxyglucose positron emission } \\ \text { tomography/computed tomography }\end{array} \\ \text { maxSUV } & \begin{array}{l}\text { Maximum standardized uptake value } \\ \text { MRI }\end{array} \\ \text { Magnetic resonance imaging } \\ \text { RFS } & \text { Recurrence-free survival } \\ \text { ROC } & \text { Receiver operating characteristic }\end{array}$

Introduction

${ }^{18} \mathrm{~F}$-fluorodeoxyglucose positron emission tomography/ computed tomography (FDG-PET/CT) is an effective and popular technique for evaluating patients before and after breast cancer surgery. This is because a single scan allows visualization of lymph node metastasis and common sites 
of breast cancer metastasis, such as the lungs, liver, and bone [1-4] and even small lesions anywhere in the body.

Quantitative FDG-PET/CT imaging is becoming prevalent in cancer treatment as it measures glucose metabolism that reflects the growth potential and metabolic activity of malignant tumors. These data cannot be obtained by morphological diagnosis using CT or magnetic resonance imaging (MRI). The FDG-PET/CT findings of primary lesions in colorectal and lung cancers correlate with metastasis and prognosis because FDG reflects tumor viability [5-7]. The technique is valuable for predicting the prognosis of patients with recurrent breast cancer and for determining and predicting the outcomes of neoadjuvant chemotherapy [8-10]. Therefore, FDG-PET/CT is useful not only for evaluating metastasis but also for predicting the prognosis of recurrent breast cancer and measuring treatment effects. However, reports remain limited to small-scale clinical trials of about 100 patients.

If the maximum standardized uptake value (maxSUV) became a prognostic factor for breast cancer, then the selection of general preoperative chemotherapy, endocrine, targeted, and localized therapies such as surgery and radiation might help to improve patient prognoses and quality of life. The present study examines the relationship between maxSUV, degree of malignancy of the primary lesion, and prognosis in two medical facilities that used different PET/CT devices.

\section{Patients and methods}

This study enrolled 344 patients with stages I-III breast cancer who underwent preoperative FDG-PET/CT and radical surgery at the Hiroshima University Department of Surgical Oncology and the Shikoku Cancer Center between January 2006 and December 2011. Patients who had undergone neoadjuvant therapy were excluded. The Institutional Review Board of each facility approved the study, and results from the database were analyzed in a retrospective multi-facility collaboration.

\section{FDG-PET/CT imaging}

The patients fasted for at least $4 \mathrm{~h}$ before being intravenously injected with $3.7 \mathrm{MBq} / \mathrm{kg}$ of $\mathrm{FDG}$, and then rested for 1-1.5 h before being evaluated. Blood glucose was measured before tracer injection to ensure a level of $150 \mathrm{mg} / \mathrm{dL}$. Patients with blood glucose values of $>150 \mathrm{mg} / \mathrm{dL}$ during PET/CT image acquisition were excluded. All patients were assessed using an integrated Discovery ST16 PET/CT (GE Healthcare, Little Chalfont, UK) or an Aquiduo (Toshiba Medical Systems Corporation, Otawara, Japan) scanner. Unenhanced CT images of 2-4-mm thick sections that matched the PET images were acquired from the head to the pelvic floor of each patient using a standard protocol. Immediately after CT, PET covered the identical axial FOV during 2-4 min per table position depending on the condition of the patient and the scanner performance. Both PET and CT studies proceeded with the patient under normal tidal breathing. All PET images were reconstructed using an iterative algorithm with CT-derived attenuation correction using Fourier re-binning followed by ordered-subset expectation maximization (iteration number 5, subset number 16). MaxSUV was established by drawing regions of interest (ROI) around the primary tumor on attenuation-corrected FDGPET images and calculated using the software integrated within the PET/CT scanner based on the formula:

$\operatorname{maxSUV}=\mathrm{C}(\mathrm{MBq} / \mathrm{kg}) /[\mathrm{ID}(\mathrm{MBq}) / \mathrm{w}(\mathrm{kg})]$,

where $\mathrm{C}$ is defined as maximal activity at a pixel within the tissue identified by ROI and ID is defined as the injected dose/kg of body weight (w). The maxSUV was adopted because values are less variable than those of mean SUV.

Histological examination

Nuclear grade was determined according to General Rules for Clinical and Pathological Recording of Breast Cancer, 17th edition [11]. The presence of estrogen (ER) and progesterone $(\mathrm{PgR})$ receptors was assessed by immunohistochemistry (IHC) and scored according to the Allred system. Human epidermal growth factor receptor-2 (HER-2) positivity was defined as $3+$ by IHC or $2+$ by gene amplification using fluorescence in situ hybridization (FISH) of $>2.2$-fold.

\section{Statistical analysis}

Data are presented as numbers $(\%)$ or as mean \pm SD unless otherwise stated. Frequencies were compared in all patients using the Chi-square test for categorical variables. Continuous variables were analyzed using a $t$ test. Recurrence was analyzed using a multivariable analysis with the Cox proportional hazards regression model. Recurrencefree survival (RFS) was defined as elapsed time from the date of surgery until the first event (relapse or death from any cause) or last follow-up. The duration of RFS was analyzed using the Kaplan-Meier method. Differences in RFS were assessed using the log rank test. Data were analyzed using the Statistical Package for the Social Sciences v. 10.5 (SPSS Inc., Chicago, IL, USA).

\section{Results}

MaxSUV, which is the most popular FDG-PET/CT value, can vary up to $30 \%$ because of differences among PET/CT 


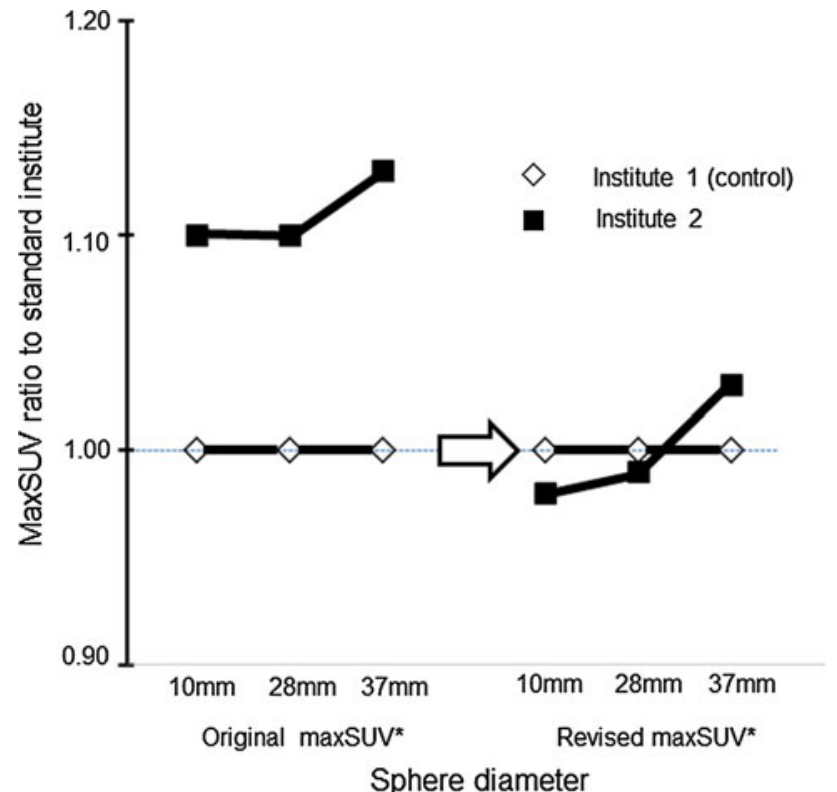

Fig. 1 MaxSUV adjusted using experimental phantom

devices and among the operators who create the images [12]. Consequently, adjustments are necessary to determine whether maxSUV is an accurate and objective indicator in multifacility collaborative studies involving different PET/CT devices. Therefore, we used a phantom to initially adjust for differences in maxSUV values determined at the two facilities (Fig. 1). The maxSUV values of the two facilities are indicated by black and white squares, respectively, and the original maxSUV values (left panel) were compared with the revised values (right panel), which were adjusted to approximately equalize the maxSUV values at both facilities.

Table 1 shows the features of the study participants. Mean maxSUV values were determined for patients grouped according to age, clinical stage (such as $\mathrm{T}$ - and $\mathrm{N}$-factors; Table 1), pathological factors (nuclear grade, ER, HER-2, and vascular invasion), and the presence or absence of recurrence (Table 2). The mean age of the study participants was $58.0 \pm 12.5$ years. The maxSUV increased with increased staging levels or degrees of malignancy, such as $\mathrm{N}$-factor, nuclear grade, and vascular invasion, and maxSUV was associated with biochemical factors such as ER and HER-2 levels.

The relationship between maxSUV and breast cancer recurrence was investigated. The revised maxSUV was used to create receiver operating characteristic (ROC) curves for recurrence (area under the curve $=0.713$ ) in which the cut-off maxSUV was 3.0 (Fig. 2). The patients were assigned to one of two groups according to the cut-off (Fig. 2), and then relationships between clinicopathological factors and maxSUV were examined (Table 3). Univariate analysis revealed correlations between maxSUV and the presence or absence of recurrence, clinical T-factor, HER-
Table 1 Patients' characteristics

\begin{tabular}{|c|c|c|c|}
\hline & $n$ & Rate $(\%)$ & $\operatorname{MaxSUV}($ mean $\pm \mathrm{SD})$ \\
\hline \multicolumn{4}{|c|}{ Clinical T-factor } \\
\hline $\mathrm{T} 1$ & 241 & 70.1 & $2.76 \pm 2.00$ \\
\hline $\mathrm{T} 2$ & 101 & 29.4 & $5.83 \pm 4.12$ \\
\hline $\mathrm{T} 3$ & 2 & 0.6 & $4.86 \pm 1.31$ \\
\hline \multicolumn{4}{|c|}{ Clinical N-factor } \\
\hline NO & 261 & 75.9 & $3.47 \pm 3.03$ \\
\hline N1 & 67 & 19.5 & $3.95 \pm 2.76$ \\
\hline $\mathrm{N} 2$ & 12 & 3.5 & $5.48 \pm 4.49$ \\
\hline N3 & 4 & 1.2 & $6.57 \pm 5.21$ \\
\hline \multicolumn{4}{|c|}{ Clinical stage } \\
\hline I & 194 & 56.4 & $2.61 \pm 1.82$ \\
\hline II & 134 & 39 & $4.96 \pm 3.71$ \\
\hline III & 16 & 4.7 & $5.76 \pm 4.70$ \\
\hline
\end{tabular}

$S D$ standard deviation

Table 2 Pathological findings

\begin{tabular}{|c|c|c|c|}
\hline & $n$ & Rate $(\%)$ & $\operatorname{MaxSUV}($ mean $\pm \mathrm{SD})$ \\
\hline \multicolumn{4}{|c|}{ Nuclear grade } \\
\hline 1 & 79 & 23 & $2.94 \pm 1.99$ \\
\hline 2 & 122 & 35.5 & $3.27 \pm 3.12$ \\
\hline 3 & 143 & 41.6 & $4.43 \pm 3.46$ \\
\hline \multicolumn{4}{|l|}{ ER } \\
\hline$(+)$ & 292 & 84.9 & $3.43 \pm 2.77$ \\
\hline$(-)$ & 52 & 15.1 & $5.01 \pm 4.40$ \\
\hline \multicolumn{4}{|l|}{$\operatorname{PgR}$} \\
\hline$(+)$ & 256 & 74.4 & $3.33 \pm 2.67$ \\
\hline$(-)$ & 88 & 25.6 & $4.68 \pm 4.00$ \\
\hline \multicolumn{4}{|c|}{ HER2 } \\
\hline$(+)$ & 47 & 13.7 & $5.01 \pm 3.56$ \\
\hline$(-)$ & 297 & 86.3 & $3.46 \pm 2.99$ \\
\hline \multicolumn{4}{|l|}{ LNM } \\
\hline$(+)$ & 110 & 32 & $4.02 \pm 2.92$ \\
\hline$(-)$ & 234 & 68 & $3.51 \pm 3.20$ \\
\hline \multicolumn{4}{|l|}{ VI } \\
\hline$(+)$ & 94 & 27.3 & $3.87 \pm 2.77$ \\
\hline$(-)$ & 250 & 72.7 & $3.60 \pm 3.24$ \\
\hline
\end{tabular}

ER estrogen receptor, HER2 human epidermal factor receptor 2, LNM lymph node metastasis, $P g R$ progesterone receptor $V I$ vascular invasion

2, nuclear grade, clinical $\mathrm{N}$-factor, $\mathrm{PgR}$, and ER ranked in ascending order based on odds ratios.

Figure 3 shows Kaplan-Meier RFS curves for maxSUV, clinical $\mathrm{T}$ - and $\mathrm{N}$-factors, nuclear grade, ER and HER-2 that are known prognostic factors for breast cancer. Recurrence-free survival significantly correlated with 


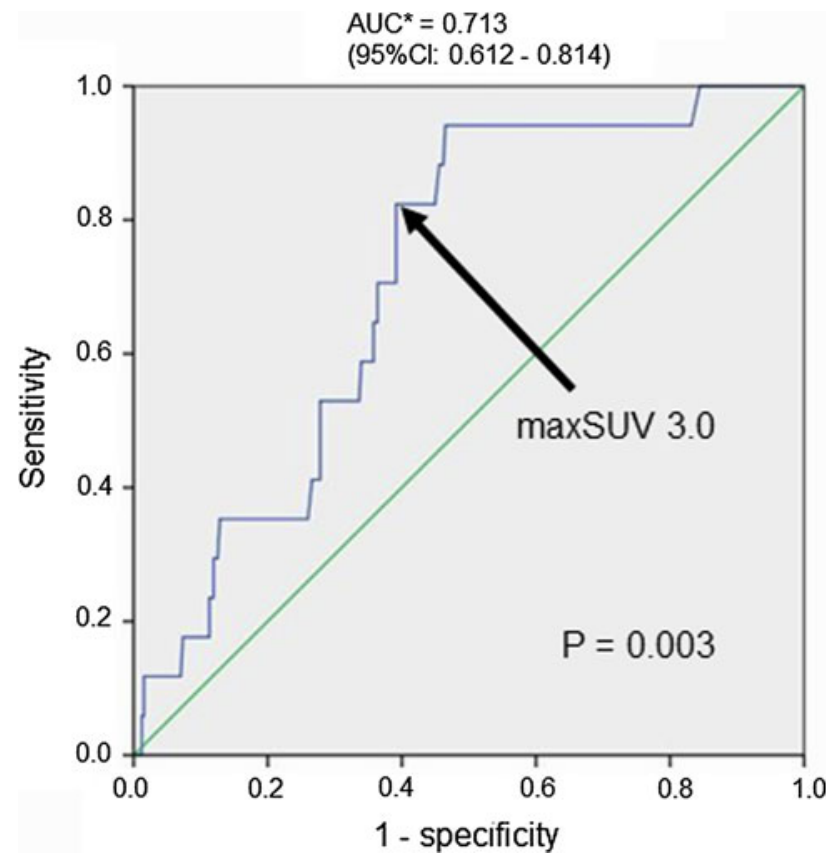

Fig. 2 Receiver operating characteristics (ROC) curves of maxSUV for recurrence in all patients $(n=344)$. Optimal cut-off of maxSUV, $3.0(n=344$, AUC: $0.713 ; 95 \%$ CI $0.612-0.814, p=0.003)$

clinical T-factor and ER, and the log-rank test showed that maxSUV also significantly correlated with recurrence $(p=0.001)$ (Fig. 3a). However, $\mathrm{N}$-factor, nuclear grade, or HER-2 did not correlate.

Univariate analysis of recurrence in all patients included the variables of age, clinical T- and N-factors, maxSUV, nuclear grade, ER, and HER-2. Both maxSUV and ER were significant prognostic factors (Table $4, p=0.004$ and $p<0.001$, respectively) and clinical T-factor and HER-2 were potentially associated with recurrence $(p<0.1)$. Multivariate analysis selected both maxSUV and ER as independent prognostic factors (Table $4, p=0.033$ and $p=0.004$, respectively).

\section{Discussion}

The prognostic factors applied in breast cancer can be broadly divided into those that determine staging and those that determine biological tumor characteristics. Factors that determine the rate of cancer progression include $\mathrm{T}$-factor (tumor diameter) and $\mathrm{N}$-factor (presence or absence/number of lymph node metastasis). Factors that determine the nature of tumors also include ER, HER-2, Ki-67 labeling index, and nuclear grade. Prognosis was previously predicted based on $\mathrm{T}, \mathrm{N}$, and $\mathrm{M}$ staging, which indicates the degree of progression. However, prognosis is now predicted and treatment regimes are presently selected by also considering ER and HER-2 levels, which determine the
Table 3 Comparison of clinicopathological parameters between $\operatorname{maxSUV} \leq 3.0$ and $>3.0$

\begin{tabular}{|c|c|c|c|c|}
\hline Variables & $\begin{array}{l}\text { MaxSUV } \\
\leq 3.0 \\
(n=199)\end{array}$ & $\begin{array}{l}\text { MaxSUV } \\
>3.0 \\
(n=145)\end{array}$ & $\begin{array}{l}\text { Odds ratio } \\
(95 \% \mathrm{CI})\end{array}$ & $p$ \\
\hline Age & $58.9 \pm 12.3$ & $56.7 \pm 12.7$ & & 0.1165 \\
\hline \multicolumn{5}{|c|}{ Clinical T-factor } \\
\hline $\mathrm{T} 1$ & 169 & 72 & 5.71 & \multirow[t]{2}{*}{$<0.0001$} \\
\hline $\mathrm{T} 2, \mathrm{~T} 3$ & 30 & 73 & $(3.44-9.48)$ & \\
\hline \multicolumn{5}{|c|}{ Clinical N-factor } \\
\hline No & 162 & 99 & 2.03 & \multirow[t]{2}{*}{0.0049} \\
\hline $\mathrm{N} 1, \mathrm{~N} 2, \mathrm{~N} 3$ & 37 & 46 & $(1.23-3.35)$ & \\
\hline \multicolumn{5}{|l|}{ Nuclear grade } \\
\hline 1,2 & 134 & 67 & 2.4 & \multirow[t]{2}{*}{$<0.0001$} \\
\hline 3 & 65 & 78 & $(1.54-3.73)$ & \\
\hline \multicolumn{5}{|l|}{ ER } \\
\hline$(+)$ & 176 & 116 & 1.91 & \multirow[t]{2}{*}{0.0309} \\
\hline$(-)$ & 23 & 29 & $(1.06-3.47)$ & \\
\hline \multicolumn{5}{|l|}{ PgR } \\
\hline$(+)$ & 159 & 97 & 1.97 & \multirow[t]{2}{*}{0.0063} \\
\hline$(-)$ & 40 & 48 & $(1.21-3.21)$ & \\
\hline \multicolumn{5}{|l|}{ HER-2 } \\
\hline$(+)$ & 182 & 115 & 2.79 & \multirow[t]{2}{*}{0.0012} \\
\hline$(+)$ & 17 & 30 & $(1.47-5.29)$ & \\
\hline \multicolumn{5}{|l|}{ LNM } \\
\hline$(-)$ & 146 & 88 & 1.78 & \multirow[t]{2}{*}{0.0128} \\
\hline$(+)$ & 53 & 57 & $(1.13-2.82)$ & \\
\hline \multicolumn{5}{|l|}{ VI } \\
\hline$(-)$ & 155 & 95 & 1.85 & \multirow[t]{2}{*}{0.011} \\
\hline$(+)$ & 44 & 50 & $(1.15-2.99)$ & \\
\hline \multicolumn{5}{|l|}{ Recurrence } \\
\hline$(-)$ & 196 & 131 & 6.98 & \multirow[t]{2}{*}{0.0014} \\
\hline$(+)$ & 3 & 14 & $(1.97-24.77)$ & \\
\hline
\end{tabular}

nature of the tumor [13-15]. Increasing the number of prognostic factors should result in more accurate and improved patient prognoses through the selection of more individualized therapies.

Although conventional CT or MRI can provide information for morphological diagnoses, FDG-PET/CT adds further information, such as that regarding tumor growth potential. In particular, maxSUV presently serves as an indicator of metabolic activity during cancer therapy. For instance, the maxSUV of primary lung and hematological cancer lesions correlates with metastasis and prognosis, whereas maxSUV also seems useful for predicting the prognosis of recurrent breast cancer and in determining and predicting the outcome of neoadjuvant chemotherapy [7, 16]. However, these reports are mainly derived only from small-scale single-facility studies, and data about sample sizes are insufficient to draw conclusions. 
Fig. 3 Recurrence-free survival (RFS) curves of patients according to predictive factors. 5-year RFS rates for patients with a maxSUV $\leq 3.0$ and $>3.0$, b clinical T-factor $\mathrm{T} 1$ and $\mathrm{T} 2,3$, c clinical $\mathrm{N}$-factors $\mathrm{N} 0$ and $\mathrm{N} 1,2,3$, d nuclear grades 1 , 2, and 3 e ER $(+)$ and ER $(-)$ and $\mathbf{f}$ HER2 $(-)$ and HER2 $(+)$
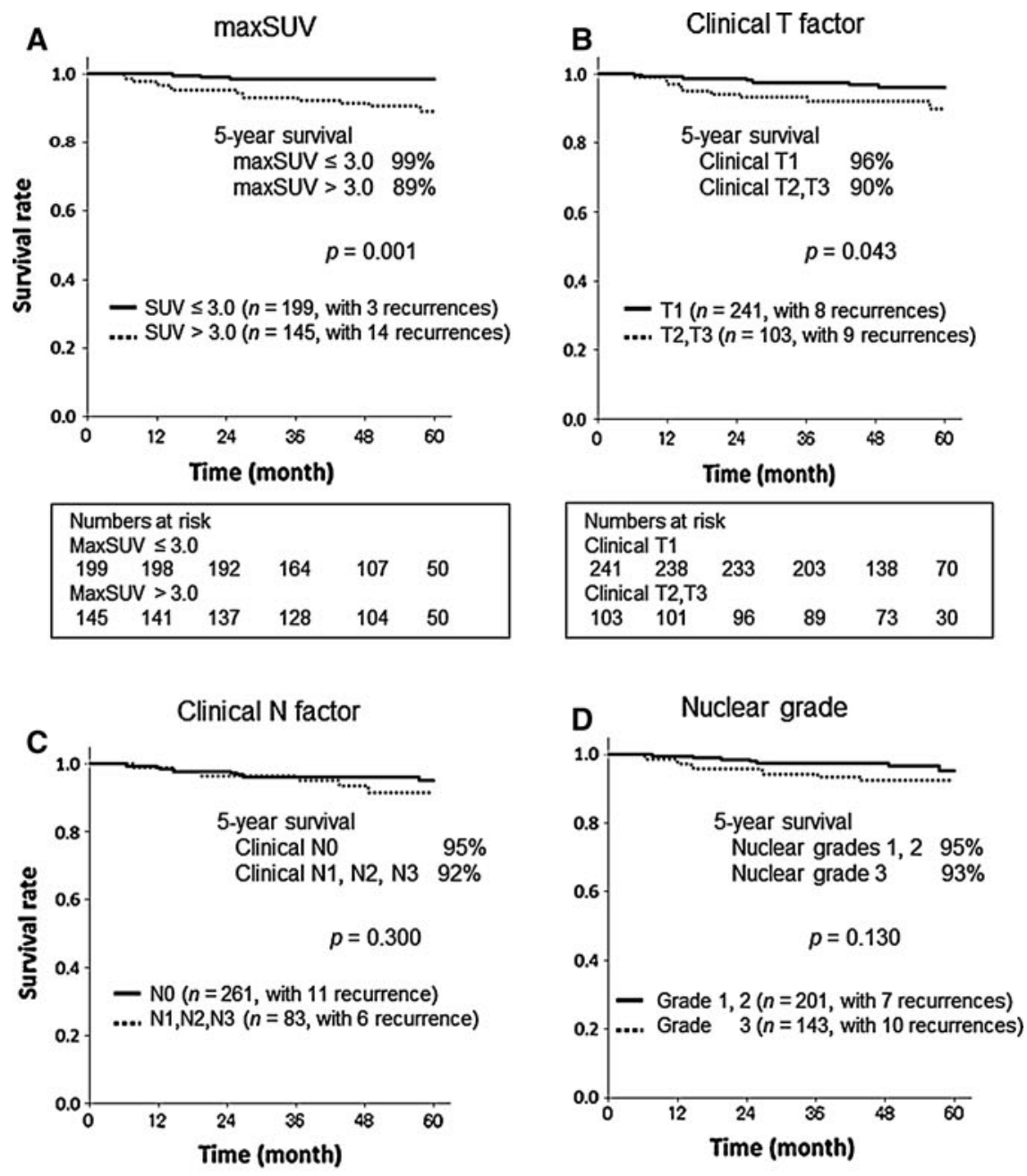

\begin{tabular}{|c|c|c|c|c|c|}
\hline \multicolumn{6}{|c|}{$\begin{array}{l}\text { Numbers at risk } \\
\text { Clinical N0 }\end{array}$} \\
\hline 261 & 257 & 250 & 223 & 161 & 72 \\
\hline \multicolumn{6}{|c|}{ Clinical N1, N2, N3 } \\
\hline 83 & 82 & 79 & 69 & 50 & 28 \\
\hline
\end{tabular}

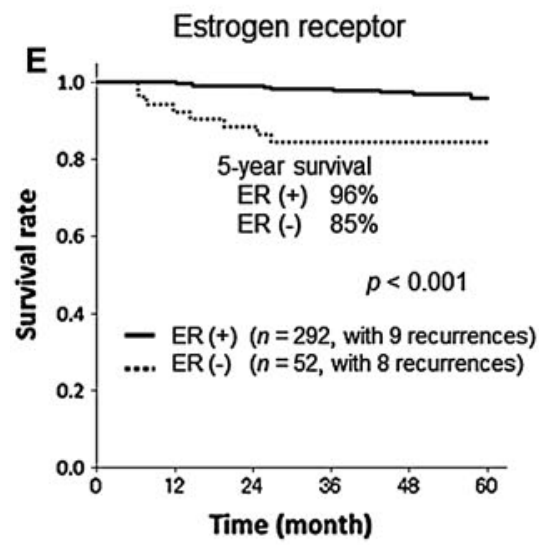

\begin{tabular}{|c|c|c|c|c|c|}
\hline \multicolumn{6}{|c|}{$\begin{array}{l}\text { Numbers at risk } \\
\mathrm{ER}(+)\end{array}$} \\
\hline $\begin{array}{l}292 \\
\text { ER (-) }\end{array}$ & 291 & 284 & 253 & 184 & 85 \\
\hline 52 & 48 & 45 & 39 & 27 & 15 \\
\hline
\end{tabular}

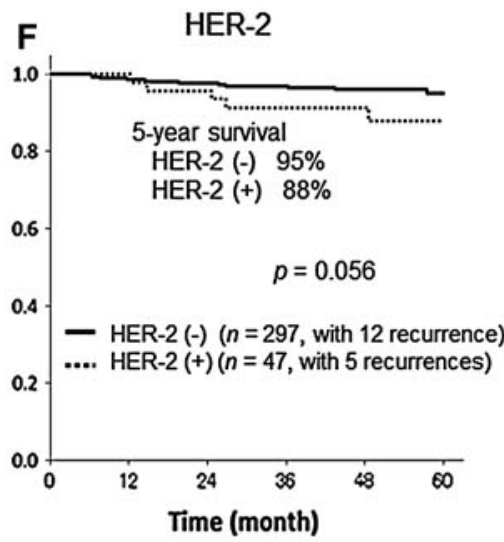

\begin{tabular}{|c|c|c|c|c|c|}
\hline \multicolumn{6}{|c|}{ Numbers at risk } \\
\hline \multicolumn{6}{|c|}{ Nuclear grade 1, 2} \\
\hline 201 & 200 & 195 & 176 & 131 & 56 \\
\hline \multicolumn{6}{|c|}{ Nuclear grade } \\
\hline 143 & 139 & 134 & 116 & 80 & 44 \\
\hline
\end{tabular}

\begin{tabular}{|c|c|c|c|c|}
\hline \multicolumn{5}{|c|}{$\begin{array}{l}\text { Numbers at risk } \\
\text { HER-2 (-) }\end{array}$} \\
\hline $\begin{array}{ll}297 & 292 \\
\text { HER-2 (+) }\end{array}$ & 285 & 251 & 180 & 88 \\
\hline 47 & 44 & 41 & 31 & 12 \\
\hline
\end{tabular}


Table 4 Univariate and multivariate analysis of recurrence using Cox model

\begin{tabular}{llllr}
\hline Factors & Favorable & Unfavorable & Hazard ratio $(95 \%$ CI $)$ & $p$ \\
\hline Univariate analysis & & & & \\
Age (year) & $<58$ & $\geq 58$ & $1.086(0.419-2.818)$ & 0.865 \\
Clinical T-factor & $\mathrm{T} 1$ & $\mathrm{~T} 2, \mathrm{~T} 3$ & $2.579(0.994-6.692)$ & 0.051 \\
Clinical N-factor & $\mathrm{N} 0$ & $\mathrm{~N} 1, \mathrm{~N} 2, \mathrm{~N} 3$ & $1.683(0.622-4.550)$ & 0.305 \\
MaxSUV & $\leq 3.0$ & $>3.0$ & $6.177(1.773-21.519)$ & 0.004 \\
Nuclear grade & 1,2 & 3 & $2.076(0.790-5.458)$ & 0.138 \\
ER & $(+)$ & $(-)$ & $5.528(2.132-14.336)$ & $<0.001$ \\
HER2 & $(-)$ & $(+)$ & $2.661(0.937-7.554)$ & 0.066 \\
Multivariate analysis & & & & \\
Age & $<58$ & $\geq 58$ & $1.338(0.496-3.607)$ & 0.565 \\
Clinical T-factor & $\mathrm{T} 1$ & $\mathrm{~T} 2, \mathrm{~T} 3$ & $1.668(0.597-4.656)$ & 0.329 \\
Clinical N-factor & $\mathrm{N} 0$ & $\mathrm{~N} 1, \mathrm{~N} 2, \mathrm{~N} 3$ & $1.183(0.418-3.344)$ & 0.751 \\
MaxSUV & $\leq 3.0$ & $>3.0$ & $4.350(1.127-16.786)$ & 0.033 \\
Nuclear grade & 1,2 & 3 & $0.955(0.332-2.745)$ & 0.932 \\
ER & $(+)$ & $(-)$ & $4.624(1.635-13.082)$ & 0.004 \\
HER2 & $(-)$ & $(+)$ & $1.247(0.407-3.824)$ & 0.699 \\
\hline
\end{tabular}

The retrospective clinical analysis at two medical facilities included 344 patients with stages I-III operable breast cancer. Chemotherapy and hormone therapy were administered at each facility, according to guidelines for case staging and biological characteristics [17, 18]. Patients who had received neoadjuvant chemotherapy were excluded to simplify the analysis, and $84.9,13.7$, and $10.2 \%$ of the patients had ER-positive, HER-2-positive, and triple-negative breast cancers, respectively. ER receptor-positive breast cancers were relatively common, whereas the odds ratios for HER-2positive breast cancers and triple-negative breast cancers were quite low.

The maxSUV cut-off calculated from ROC curves for recurrence was 3.0, and when the patients were divided based on this cut-off, maxSUV significantly correlated with almost all clinicopathological factors for breast cancer. Because of the large number of clinicopathological factors for breast cancer such as tumor diameter, lymph node metastasis ER, and HER-2, we assumed that maxSUV would be the ultimate output of these factors. For example, the degree of malignancy would increase with an increase in maxSUV when ER or HER-2 signaling is involved.

Univariate and multivariate analyses identified both ER status and maxSUV as independent prognostic factors. Furthermore, since clinical $\mathrm{T}$ - and $\mathrm{N}$-factors, nuclear grade, and HER-2 were not included as prognostic factors in our analyses, we can state that maxSUV has a closer correlation with prognosis than these factors. Moreover, RFS was assessed using Kaplan-Meier curves and the log-rank test, which revealed that maxSUV, clinical T-factor and ER were significant prognostic factors, as noted above. Statistical analysis of RFS and multivariate analysis demonstrated that
maxSUV was a useful novel prognostic factor for breast cancer in our patients.

Our results showed that maxSUV has the potential to be a novel prognostic factor and that it can be used to determine future therapies. However, different courses of treatment are currently prescribed for each biological characteristic (subtype). Thus, the distribution and characteristics of the maxSUV must be known before courses of treatment can be determined. The maxSUV was the highest for the triplenegative type among all of the subtypes [19]. In the future, steps are needed to determine the cut-offs for each subtype in more patients including those with luminal, HER-2 and triplenegative breast cancers, to determine courses of treatment.

Our results indicate that maxSUV could serve as a new prognostic factor for breast cancer, but more data should be accumulated from more patients. Moreover, maxSUV might be used as a factor to predict the effects of chemotherapy and endocrine therapy.

Conflict of interest The authors declare that they have no conflicts of interest.

Open Access This article is distributed under the terms of the Creative Commons Attribution Noncommercial License which permits any noncommercial use, distribution, and reproduction in any medium, provided the original author(s) and the source are credited.

\section{References}

1. Koolen BB, Vrancken Peeters MJ, Aukema TS et al (2012) 18FFDG PET/CT as a staging procedure in primary stage II and III breast cancer: comparison with conventional imaging techniques. Breast Cancer Res Treat 131:117-126 
2. Riegger C, Herrmann J, Nagarajah J et al (2012) Whole-body FDG PET/CT is more accurate than conventional imaging for staging primary breast cancer patients. Eur J Nucl Med Mol Imaging 39:852-863

3. Ohsumi S, Inoue T, Kiyoto S, Hara F et al (2011) Detection of isolated ipsilateral regional lymph node recurrences by F18fluorodeoxyglucose positron emission tomography-CT in followup of postoperative breast cancer patients. Breast Cancer Res Treat 130:267-272

4. Kurata A, Murata Y, Kubota K et al (2009) Multiple (18)F-FDG, PET-CT for postoperative monitoring of breast cancer patients. Acta Radiol 50:979-983

5. Dimitrakopoulou-Strauss A, Strauss LG et al (2004) Prognostic aspects of $18 \mathrm{~F}-\mathrm{FDG}$ PET kinetics in patients with metastatic colorectal carcinoma receiving FOLFOX chemotherapy. J Nucl Med 45:1480-1487

6. Tsutani Y, Takuwa T, Miyata Y et al (2013) Prognostic significance of metabolic response by positron emission tomography after neoadjuvant chemotherapy for resectable malignant pleural mesothelioma. Ann Oncol 24:1005-1010

7. Tsutani Y, Miyata Y, Misumi K et al (2011) Difference in prognostic significance of maximum standardized uptake value on [18F]-fluoro-2-deoxyglucose positron emission tomography between adenocarcinoma and squamous cell carcinoma of the lung. Jpn J Clin Oncol 41:890-896

8. Morris PG, Ulaner GA, Eaton A et al (2012) Standardized uptake value by positron emission tomography/computed tomography as a prognostic variable in metastatic breast cancer. Cancer 118:5454-5462

9. Straver ME, Aukema TS, Olmos RA et al (2010) Feasibility of FDG PET/CT to monitor the response of axillary lymph node metastases to neoadjuvant chemotherapy in breast cancer patients. Eur J Nucl Med Mol Imaging 37:1069-1076

10. Wang Y, Zhang C, Liu J et al (2012) Is 18F-FDG PET accurate to predict neoadjuvant therapy response in breast cancer? A metaanalysis. Breast Cancer Res Treat 131:357-369
11. The Japanese Breast Cancer Society (2012) General rules for clinical and pathological recording of breast cancer, 17th edn. Kanehara, Tokyo

12. Westerterp M, Pruim J, Oyen W et al (2007) Quantification of FDG PET studies using standardised uptake values in multicentre trials: effects of image reconstruction, resolution and ROI definition parameters. Eur J Nucl Med Mol Imaging 34:392-404

13. Wang Y, Yin Q, Yu Q et al (2011) A retrospective study of breast cancer subtypes: the risk of relapse and the relations with treatments. Breast Cancer Res Treat 130:489-498

14. Munzone E, Botteri E, Sandri MT et al (2012) Prognostic value of circulating tumor cells according to immunohistochemically defined molecular subtypes in advanced breast cancer. Clin Breast Cancer 12:340-346

15. Goldhirsch A, Wood WC, Coates AS et al (2011) Strategies for subtypes - dealing with the diversity of breast cancer: highlights of the St. Gallen International Expert Consensus on the Primary Therapy of Early Breast Cancer 2011. Ann Oncol 22:1736-1747

16. Safar V, Dupuis J, Itti E et al (2012) Interim [18F] fluorodeoxyglucose positron emission tomography scan in diffuse large B-cell lymphoma treated with anthracycline-based chemotherapy plus rituximab. J Clin Oncol 30:184-190

17. Goldhirsch A, Coates AS, Gelber RD et al (2006) First-select the target: better choice of adjuvant treatments for breast cancer patients. Ann Oncol 12:1772-1776

18. Goldhirsch A, Ingle JN, Gelber RD et al (2009) Thresholds for therapies: highlights of the St Gallen International Expert Consensus on the primary therapy of early breast cancer 2009. Ann Oncol 8:1319-1329

19. Ohara M, Shigematsu H, Tsutani Y et al (2013) Role of FDG$\mathrm{PET} / \mathrm{CT}$ in evaluating surgical outcomes of operable breast cancer-usefulness for malignant grade of triple-negative breast cancer. Breast. doi:S0960-9776(13)00106-9 